\title{
artigo
}

Medeiros, T.H.R.S.; Monteiro, S.C.M.; Lima Júnior, J.R.M.; Alves, A.K.B.F.; Sampaio, T.N.S.; Teles, B.E.M.;

Fenótipo de cintura hipertrigliceridêmica e lesão renal em pacientes hipertensos

\section{Fenótipo de cintura hipertrigliceridêmica e lesão renal em pacientes hipertensos}

\author{
Hypertriglycheridemic waist phenotype and kidney injury in hypertensive patients \\ Fenotipo de cintura hipertrigliceridémica y lesión renal en pacientes hipertensos
}

\begin{abstract}
RESUMO
Objetivo: Verificar a associação entre o fenótipo de cintura hipertrigliceridêmica (FCH) e a estimativa da taxa de filtração glomerular (eTFG) em pacientes hipertensos. Método: Tratou-se de um estudo transversal e retrospectivo, composto por 146 prontuários de hipertensos consultados entre os anos de 2013 a 2017 no Centro de Prevenção de Doenças Renais HUUFMA. Considerou-se dados sociodemográficos, condições clínicas e exames laboratoriais, estes foram analisados pelo programa estatístico SPSS $21.0{ }^{\circledR}$. Resultados: 0 FCH esteve presente em $84,2 \%(n=123)$ da amostra e $65 \%(n=$, desta, eram mulheres. A eRFG apresentou média de 69,3 $\pm 23,9 \mathrm{~mL} / \mathrm{min}$ em 1,73 $\mathrm{m}^{2}$ no grupo com FCH e média de 91,4 $\pm 42,6 \mathrm{~mL} / \mathrm{min}$ em 1,73 m² no grupo sem $F C H$, com associação significativa $(p<0,05)$. Conclusões: A presença do $F H C$ associou-se à diminuição da eTFG, ao aumento dos exames laboratoriais, medidas antropométricas e índices antropométricos.
\end{abstract}

DESCRITORES: Cintura hipertrigliceridêmica; Insuficiência Renal Crônica; Obesidade abdominal; Hipertensão..

\section{ABSTRACT}

Objective: To verify the association between the hypertriglyceridemic waist phenotype (FCH) and the estimate of the glomerular filtration rate (eTFG) in hypertensive patients. Method: This was a cross-sectional and retrospective study, consisting of 146 medical records of hypertensive adults consulted between 2013 and 2017 at the HUUFMA Kidney Disease Prevention Center. Sociodemographic data, clinical conditions and laboratory tests were considered, these were analyzed by the statistical program SPSS $21.0^{\circledR}$. Results: FCH was present in $84.2 \%(n=123)$ of the sample and $65 \%(n=$, of this, were women. The eRFG presented an average of $69.3 \pm 23.9 \mathrm{~mL} / \mathrm{min}$ in $1.73 \mathrm{~m}^{2}$ in the group with FCH and a mean of $91.4 \pm 42.6 \mathrm{~mL} / \mathrm{min}$ in $1.73 \mathrm{~m}^{2}$ in the group without $F C H$, with a significant association $(p<0.05)$. Conclusions: The presence of $F H C$ was associated with decreased eTFG, the increase in laboratory tests, anthropometric measurements and anthropometric indices.

DESCRIPTORS: Hypertriglyceridemic waist ; Chronic Kidney Failure; Abdominal obesity; Hypertension.

\section{RESUMEN}

Objetivo: Verificar la asociación entre el fenotipo de cintura hipertrigliceridémica (FCH) y la estimación de la tasa de filtración glomerular (eTFG) en pacientes hipertensos. Método: Se trata de un estudio transversal y retrospectivo, que consta de 146 historias clínicas de adultos hipertensos consultados entre 2013 y 2017 en el Centro de Prevención de Enfermedades Renales HUUFMA. Se consideraron datos sociodemográficos, condiciones clínicas y pruebas de laboratorio, estos fueron analizados por el programa estadístico SPSS $21.0^{\circledR}$. Resultados: El FCH estuvo presente en el $84,2 \%(n=123)$ de la muestra y el $65 \%(n=$, de este, fueron mujeres. El eRFG presentó un promedio de $69,3 \pm 23,9 \mathrm{~mL} / \mathrm{min}$ en $1,73 \mathrm{~m}^{2}$ en el grupo con $\mathrm{FCH}$ y una media de $91,4 \pm 42,6 \mathrm{~mL} / \mathrm{min}$ en $1,73 \mathrm{~m}^{2}$ en el grupo $\sin \mathrm{FCH}$, con una asociación significativa $(p<0,05)$. Conclusiones: La presencia de $F H C$ se asoció con disminución de eTFG, aumento de pruebas de laboratorio, medidas antropométricas y antropométricas índices.

DESCRIPTORES: Cintura hipertrigliceridémica; Insuficiencia renal crónica; Obesidad abdominal; Hipertensión.

RECEBIDO EM: 28/02/2021 APROVADO EM: 15/03/2021

\section{Thaynara Helena Ribeiro e Silva Medeiros}

Mestre em Saúde do Adulto e da Criança PPGSAC- UFMA. Especialista em Nutrição Clínica- UNESA. Especialista em Nutrição Esportiva- UNESA. Graduação em Nutrição- Universidade Federal do Maranhão.

ORCID: 0000-0003-2027-5530 


\section{Sally Cristina Moutinho Monteiro}

Farmacêutica-Bioquímica. Mestrado (2001) e Doutorado (2005) em Biociências e Biotecnologia Aplicada a Farmácia pela Universidade Estadual Paulista Júlio de Mesquita Filho. Especialista em Farmácia Clínica pela Associação dos Farmacêuticos de Ribeirão Preto/SP (2010). Professora Associada do Departamento de Farmácia da Universidade Federal do Maranhão (UFMA), Professora do Programa de Pós-Graduação em Saúde do Adulto (PPGSAD) da UFMA, Pesquisadora do Banco de Tumor e DNA do Maranhão (BTMA) - UFMA e Membro do PET-IP UFMA, São Luís.

ORCID: 0000-0002-4425-1552

\section{José de Ribamar Medeiros Lima Júnior}

Mestre em Enfermagem (Programa de Pós- graduação em Enfermagem- UFMA). Especialista em UTI Pediátrica - Faculdade Cidade Verde. Graduação em Enfermagem- Universidade Federal do Maranhão.

ORCID: 0000-0001-9172-3682

\section{Adriana Karina Beckman Ferreira Alves}

Graduação em Nutrição- Faculdade Santa Terezinha (CEST). Especialista em Terapia Nutricional Enteral e Parenteral -GANEP. ORCID: 0000-0001-7069-0586

\section{Thaynara Nascimento da Silva Sampaio}

Graduação em Nutrição- Faculdade Estácio de Sá de São Luís.

ORCID: 0000-0003-1133-1633

\section{Brenda Emmylly Marinho Teles}

Graduação em Nutrição- Faculdade Estácio de Sá de São Luís. ORCID: 0000-0001-5236-5691

\section{INTRODUÇÃO}

A insuficiência renal aguda (IRA) é uma patologia reversível, ou seja, tem forte potencial de regresso da doença, é caracterizada por um rápido declínio na capacidade dos rins de remover a escória corporal ${ }^{1}$. Suas manifestações mais comuns são sede, hipotensão ortostática, redução da pressão venosa jugular, sinais de desidratação, alterações nos resultados do sedimento urinário, edema, hipertensão e oligúria ${ }^{2}$. Em geral, as indicações para reversão da IRA são terapia renal substitutiva (TRS) ou diálise $e^{2}$. Esse tratamento é o mais utilizado em unidade de terapia intensiva (UTI), devendo ser iniciado em situações agudas diante da sobrecarga de toxinas ou hipervolemia grave que pode resultar em complicações ou danos a outros órgãos ${ }^{3}$.

A insuficiência renal crônica (DRC) ou doença renal crônica (DRC) é um termo usado para indivíduos com alterações heterogêneas que afetam tanto a função renal quanto a função, com múltiplas causas e múltiplos fatores prognósticos ${ }^{4}$. É uma doença insidiosa de longo prazo que, na maior parte de sua evolução, é assintomá- tica ${ }^{3,4}$. Essa condição clínica é caracterizada pela retenção de toxinas urêmicas na pele, associada à perda irreversível da função renal. Sabe-se que as toxinas urêmicas afetam várias partes do corpo, incluindo o cérebro e, consequentemente, levam à neuropatia periférica e à disfunção do $\mathrm{SNC}^{5}$.

$\mathrm{O}$ aumento do número de casos tem sido relatado na última década em diferentes contextos associados ao envelhecimento e transição demográfica da populaçãa ${ }^{5,6}$. As principais causas de dano renal são hipertensão arterial sistêmica, diabetes mellitus, glomerulonefrite, idade avançada, excesso de peso, tabagismo, dislipidemias e uso de drogas nefrotóxicas, enquanto as disparidades socioeconômicas, raciais e de gênero também são fatores contribuintes. No Brasil, mais de $20 \%$ dos adultos têm hipertensão arterial sistêmica (HAS), 8\% de diabetes mellitus (DM), $18 \%$ são fumantes e $50 \%$ apresentam excesso de peso ${ }^{3,7,8}$.

Apesar do tratamento, a mortalidade é maior no início da terapia devido ao diagnóstico tardio. Por esses motivos, a adoção de medidas preventivas de lesão renal, como a identificação de fatores de risco e grupos, ou medidas que facilitam o diag- nóstico precoce, têm recebido atenção e

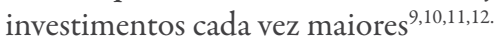

Além dos métodos de triagem convencionais, é importante encontrar métodos alternativos, de baixo custo e alta população. Nesse contexto, o Fenótipo Cintura Hipertrigliceridêmica $(\mathrm{FCH})$, que envolve a mensuração de medidas simples, de baixo custo, facilmente aplicáveis à saúde pública e clínica, como a circunferência da cintura e triglicerídeos (TG) sendo simultaneamente aumentado) demonstra ser uma ferramenta de triagem ideal para detectar indivíduos em risco de diabetes tipo 2, doenças cardiovasculares e doença renal crônica.

O fenótipo de cintura hipertrigliceridêmica (FHC) foi proposto pela primeira vez por Lemieux et al. (2000); sendo definida como uma situação simultânea de elevação da circunferência da cintura (CC) e aumento dos valores de triglicerídeos plasmáticos. A avaliação da cintura hipertrigliceridêmica é simples, de baixo custo, não invasiva e pode auxiliar na identificação do excesso de tecido adiposo visceral e predizer alterações cardiovasculares e metabólicas, contribuindo como uma ferramenta para abordagem na prática clínica ${ }^{9,10,11}$. 
Nesse contexto, se faz necessário conhecer a associação entre a presença do fenótipo de cintura hipertrigliceridêmica (FHC) com o aparecimento de lesão renal, a fim de minimizar risco e possíveis efeitos adversos aos pacientes acometidos.

\section{MÉTODOS}

Estudo com delineamento transversal, retrospectivo e de abordagem quantitativa, composto por amostra de conveniência, realizado pela análise dos dados dos prontuários decorrentes de consultas de indivíduos com hipertensão arterial (previamente diagnosticados) acompanhados a nível ambulatorial pela liga acadêmica de hipertensão da Universidade Federal do Maranhão (UFMA) no Centro de Prevenção de Doença Renal (CPDR), no Hospital Universitário Presidente Dutra (HUPD). Este estudo faz parte de um Projeto maior intitulado "Predição de doença renal crônica através de redes neurais inteligentes", o qual possui aprovação do Comitê de Ética em Pesquisa do Hospital Universitário Presidente Dutra (CEP-HU-UFMA), sob o no $2.035 .753 \mathrm{e}$ CAAE 67030517.5.0000.5087.

As bases de dados utilizadas para o estudo foram Medline, Lilacs, SciELO, usando os seguintes descritores: Hypertriglyceridemic waist. Chronic Kidney Failure. Abdominal obesity. Hypertension.

A coleta foi realizada durante os meses de novembro de 2017 a abril de 2018 . Foram incluídos indivíduos de ambos os sexos, sem distinção de etnia, adultos e idosos, que foram consultados no ambulatório do Centro de Prevenção de Doença Renal, entre os anos de 2013 a 2107. Foram excluídos todos aqueles com histórico de doenças consumptivas, doença renal preexistente, gestantes e prontuários com preenchimento incompleto de dados.

Inicialmente foram coletados dados de 439 prontuários e após seleção segundo o período pretendido (2013 a 2017), obteve-se 203 prontuários. Após essa etapa, foram excluídos do estudo aqueles prontuários sem informações necessárias para pesquisa tais como ausência de resultados de exames laboratoriais e/ou medidas antropométricas, perfazendo uma amostragem final de 146 prontuários.

Para as variáveis sociodemográficas foi considerado o sexo (masculino e feminino), grupo etário (adultos e idosos), nível de escolaridade (superior completo, médio completo, fundamental completo e fundamental incompleto), cor autorreferida (preta ou não preta), situação conjugal (casado/união estável ou separado/ divorciado/solteiro/viúvo) e renda (até 1 salário, de mais de 1 salário a 3 salários, mais de 3 salários a 5 salários e mais que 5 salários mínimos) segundo estratificação de renda do IBGE ${ }^{12}$.

Para caracterizar os hábitos de vida, foram verificados, nos prontuários, a prática de atividade física, tabagismo e etilismo. Segundo a atividade física adotou-se a classificação de ativo (atividade física vigorosa $\geq 60$ minutos/semana; atividade física moderada, caminhada ou a soma de todas elas $\geq 150$ minutos/semana) e inativo (atividade vigorosa $<60$ minutos/semana; outras atividades menos intensas $<150$ minutos/semana) ${ }^{13}$.

Nos dois grupos de hipertensos (com fenótipo de cintura hipertrigliceridêmica e sem fenótipo) foi verificado também a presença de comorbidades, constantes no prontuário, autorreferidas tais como diabetes mellitus, hipercolesterolemia, hipo ou hipertireoidismo, doença arterial coronariana, doenças cardiovasculares autorreferidas: infarto do miocárdio, acidente vascular encefálico e histórico familiar (pai, mãe e irmãos) de diabetes mellitus, hipertensão arterial, infarto do miocárdio, acidente vascular cerebral, doença renal.

As medidas antropométricas utilizadas foram peso, altura e circunferência da cintura (CC). Os índices antropométricos avaliados foram: relação cintura estatura (RCE), índice de conicidade (IC), índice de adiposidade central (IAC) e o índice de massa corporal (IMC). Em todas as medias e índices foram comparados segundo classificação proposta pela OMS.

Foram analisados os resultados dos exames laboratoriais de glicemia em jejum, colesterol total, HDL colesterol
(HDL-c), LDL colesterol (LDL-c) e triglicerídeos. Foi calculado os índices TyG (triglicerídeos/glicose), IAP (índice de aterogenicidade plasmática) e o LAP (lipid accumulation product), frações lipídicas colesterol não HDL, relação LDL-c/ HDL-c e colesterol total/HDL-c. Além disso, também foram utilizados os valores da creatinina e uréia séricas.

A equação de Cockcroft-Gault [(140-idade $) \times$ peso/ $(72 \times$ creatinina $) \times$ 0,85 (se mulher)] foi utilizada para a estimativa do ritmo de filtração glomerular. $\mathrm{Na}$ análise em corte transversal, o ponto de corte para lesão renal foi definida como taxa de filtração glomerular de $<90 \mathrm{~mL} / \mathrm{min}$ por $1,73 \mathrm{~m}^{2}$ e para a análise em perspectiva, incidente DRC era uma taxa de filtração glomerular de $<60 \mathrm{~mL} / \min$ por $1,73 \mathrm{~m}^{2} .^{214}$

FCH variável dependente do estudo, é um indicador composto por duas variáveis: circunferência da cintura e a concentração sérica dos triglicerídeos. Para classificação da circunferência da cintura foram utilizados os pontos de corte baseados na International Diabetes Federation (IDF, 2006): Adequado: $<80 \mathrm{~cm}$ para mulheres; < $90 \mathrm{~cm}$ para homens; Inadequado: $\geq 80 \mathrm{~cm}$ para mulheres; $\geq 90 \mathrm{~cm}$ para homens 15,16 e para classificação do valor dos triglicérides: Adequado: $<150$ $\mathrm{mg} / \mathrm{dL}$, aumentado: $\geq 150 \mathrm{mg} / \mathrm{dL} 15$.

Os indivíduos que apresentaram as duas variáveis aumentadas simultaneamente foram classificados no grupo com Fenótipo de Cintura Hipertrigliceridêmica $(\mathrm{FCH})$, e caso apresentassem apenas uma dessas variáveis alteradas ou nenhuma das duas, foram incluídos no grupo sem esse fenótipo. Ambos os grupos também foram caracterizados segundo dados sociodemográficos, antropométricos, bioquímicos, condições de saúde e hábitos de vida.

Os dados obtidos foram analisados a partir do programa estatístico SPSS $21.0^{\circ}$. As variáveis numéricas foram apresentadas em médias e desvio-padrão e as variáveis categóricas foram apresentadas em frequências absolutas (n) e relativas (\%). Para relacionar as variáveis séricas e antropométricas com alterações na estimativa da Taxa de Filtração Glomerular (eTFG), foi 
aplicado o teste $\mathrm{t}$ de studdent. Utilizou-se o teste qui-quadrado para as variáveis nominais. A normalidade foi verificada através de teste de Shapiro-Wilk. Foram consideradas significativas as diferenças quando valor de $\mathrm{p}<0,05$.

\section{RESULTADOS}

Do total de 146 prontuários selecionados, foram verificados que 123 prontuários $(84,2 \%)$ apresentaram o Fenótipo da Cintura Hipertrigiceridêmica (FCH) e 23 prontuários $(15,8 \%)$ não apresentaram o fenótipo. Entre os que apresentaram o $\mathrm{FCH}$, predominou o sexo feminino (65\%), e no grupo sem fenótipo predominou o sexo masculino (52,2\%). Em ambos os grupos houve predominância de idosos com frequência na amostra total de $57,5 \%$ (Tabela 1).

Segundo a situação conjugal, $71,5 \%$ do grupo com fenótipo declararam viver com companheiro e 60,9\% do grupo sem fenótipo vivem sem companheiro. Dos prontuários que tinham informação sobre escolaridade, o grupo com $\mathrm{FCH}$ teve mais analfabetos $(32,5 \%)$ e viviam com até 1 salário $(16,3 \%)$ e no grupo sem fenótipo maioria com o ensino fundamental $(56,5 \%)$ e viviam com renda de 1 a 3 salários (52,2\%). No grupo com fenótipo $39,8 \%$ não informaram sua cor e $37,4 \%$ declararam cor não preta e no grupo sem FCH 52,2\% também declaram ser de cor não preta (Tabela 1).

Em ambos os grupos, a não prática de atividade física foi a maioria encontra- da, com $64,2 \%$ no grupo com FCH e 69,6\% no grupo sem FCH. O tabagismo também não foi referido na maioria dos prontuários de ambos os grupos, sendo $94,3 \%$ do grupo com fenótipo e $65,2 \%$ do grupo sem fenótipo. O etilismo foi referido somente em 9,8\% dos pacientes com fenótipo e em $60,9 \%$ dos pacientes sem o fenótipo (Tabela 1).

De acordo com a Tabela 2, de todos os 146 participantes a maioria $(69,2 \%)$ não tem o diagnóstico de diabetes mellitus e; dentre o grupo com $\mathrm{FCH}, 33,3 \%$ eram diabéticos, enquanto no grupo sem $\mathrm{FCH}$ o percentual de diabetes foi de $17,4 \%$. A situação de hipotireoidismo ou hipertireoidismo foi detectada somente no grupo com fenótipo, e para essa condição obteve-se percentual de $7,3 \%$ do total desse grupo.

Tabela 1. Caracterização sociodemográfica e de estilo de vida em pacientes com hipertensão arterial assistidos em um centro de prevenção de doença renal segundo presença do fenótipo da cintura hipertrigliceridêmica. São Luís, Maranhão, 2018

\section{TOTAL}

VARIÁVEIS

Sexo

Feminino

Masculino

Idade

Idoso

Adultos

Estado civil

Com companheiro

Sem companheiro

Escolaridade

Não informado

Analfabeto

Ens. fundamental

Ensino médio

Renda

Não informado

Até 1 salário

$>1$ a 3 salários

> 3 a 5 salários

> 5 a 15 salários

55

84

97

49

55

47

38

N $\quad \%$

N

SIM

\section{FENÓTIPO}

N

M

62,3

80

65

11

47,8

0,11

2,44

37,7

43

35

12

52,2

57,5

63

56,1

15

65,2

0.41

0.65

54

43,9

4

34,8

66,4

88

71,5

9

39,1

0,00

9,13

33,6

35

28,5

60,9

37,7

52

42,3

13,0

32,2

40

32,5

20,3

30,4

0.00

9.98

26,0

25

56,5

0,0

$X^{2}$

44

\section{5,5}

17,8

17,1

8,9

0,7
4,9

6

79

64,2

16,3

10,6

8,1

8

13
8,7

26,1

52,2

0.00

9.98
12

13,0

0,0 


\section{artigo}

Medeiros, T.H.R.S.; Monteiro, S.C.M.; Lima Júnior, J.R.M.; Alves, A.K.B.F.; Sampaio, T.N.S.; Teles, B.E.M.

Fenótipo de cintura hipertrigliceridêmica e lesão renal em pacientes hipertensos

\begin{tabular}{|c|c|c|c|c|c|c|c|c|}
\hline \multicolumn{9}{|l|}{ Cor } \\
\hline Não informado & 49 & 33,6 & 49 & 39,8 & 0 & 0,0 & \multirow{3}{*}{0,00} & \multirow{3}{*}{0,11} \\
\hline Preta & 39 & 26,7 & 28 & 22,8 & 11 & 47,8 & & \\
\hline Não preta & 58 & 39,7 & 46 & 37,4 & 12 & 52,2 & & \\
\hline \multicolumn{9}{|l|}{ Atividade física } \\
\hline Não & 91 & 65,1 & 79 & 64,2 & 16 & 69,6 & \multirow[t]{2}{*}{0.62} & \multirow[t]{2}{*}{0,24} \\
\hline Sim & 51 & 34,9 & 44 & 35,8 & 7 & 30,4 & & \\
\hline \multicolumn{9}{|l|}{ Tabagismo } \\
\hline Não & 131 & 89,7 & 116 & 94,3 & 15 & 65,2 & \multirow[t]{2}{*}{0,00} & \multirow[t]{2}{*}{17,78} \\
\hline Sim & 15 & 10,3 & 7 & 5,7 & 8 & 34,8 & & \\
\hline \multicolumn{9}{|l|}{ Etilismo } \\
\hline Não & 120 & 82,2 & 111 & 90,2 & 9 & 39,1 & \multirow[t]{3}{*}{0,00} & \multirow[t]{3}{*}{13,57} \\
\hline Sim & 26 & 17,8 & 12 & 9,8 & 14 & 60,9 & & \\
\hline TOTAL & 146 & 100 & 123 & 84,2 & 23 & 15,8 & & \\
\hline
\end{tabular}

Tabela 2. Condições clínicas de participantes hipertensos assistidos em um centro de prevenção de doença renal, segundo presença de fenótipo, São Luís, Maranhão, 2018.

TOTAL

VARIÁVEIS

\begin{tabular}{ccccc} 
& \multicolumn{3}{c}{ SIM } & \multicolumn{2}{c}{ NÃO } \\
N & $\%$ & N & $\%$ & N
\end{tabular}

Diabetes mellitus

Não

Sim

Hipercolesterolemia

Não

Sim

Hipo ou Hiper

Não

Sim

DAC

Não

$\operatorname{Sim}$

AVC

Não

Sim

IAM

Não

Sim

Histórico Familiar de DM

Não

Sim

Histórico Familiar de HAS
101

69,2

82

66,

45

30,8

41

33,3

19

82,6

0,12

2,30

103

70,5

80

65,0

4

17,4

43

29,5

43

35,0

137

93,8

114

92,7

23

100

0,18

1,79

9

6,2

7,3

0

0

143

97$$
3
$$

2,1

$$
145
$$

1

99

122

99,2

97,6

23

100

0,44

0,57

0,7

144

98

$2 \quad 1,4$

119

$81,5 \quad 103$

83,7

27

18,5

20
2,4

0

0

100

0,66

0,18

0

100

0,53

0,37

0

69,6

0,10

2,58 


$\begin{array}{lcccccccc}\text { Não } & 111 & 76,0 & 95 & 77,2 & 16 & 69,6 & 0,42 & 0,62 \\ \text { Sim } & 35 & 24,0 & 28 & 28,8 & 7 & 30,4 & & 3,63 \\ \text { Histórico familiar de DCV } & 142 & 97,3 & 121 & 98,4 & 21 & 91,3 & 0,05 & 0,22 \\ \text { Não } & 4 & 2,7 & 2 & 1,6 & 2 & 8,7 & & 0,37 \\ \text { Sim } & & & & & & & & \\ \text { Histórico familiar de AVC } & 122 & 83,6 & 102 & 82,9 & 20 & 87,0 & 0,63 & \\ \text { Não } & 24 & 16,4 & 21 & 17,1 & 3 & 13,0 & & \\ \text { Sim } & 144 & 98,6 & 121 & 98,4 & 23 & 100 & 0,53 & \\ \text { Histórico familiar de doença renal } & & & & & & & \\ \text { Não } & 2 & 1,4 & 2 & 1,6 & 0 & 0 & & \\ \text { Sim } & 136 & 93,2 & 113 & 91,9 & 23 & 100 & 0,15 & \\ \text { Histórico familiar de IAM } & 10 & 6,8 & 10 & 8,1 & 0 & 0 & & \\ \text { Não } & 146 & 100 & 123 & 84,2 & 23 & 15,8 & & \\ \text { Sim } & & & & & & & \end{array}$

A situação de hipercolesterolemia, doença arterial coronariana (DAC), acidente vascular cerebral (AVC) e infarto agudo do miocárdio (IAM) só foram detectados no grupo com o $\mathrm{FCH}$.

Em relação ao histórico familiar de doenças crônicas, verificou-se que somente $18,5 \%$ dos indivíduos participantes possuem histórico familiar de diabetes mellitus, sendo as pessoas pertencentes ao grupo sem FCH obtiveram percentual de $30,4 \%$ e os participantes com FCH obtiveram $16,3 \%$ de percentual. Para o histórico familiar de hipertensão arterial, somente $24 \%$ da amostra apresentou resposta positiva, sendo $30,4 \%$ dos indivíduos sem fenótipo e 22,8\% do grupo com fenótipo. Para a presença de doença cardiovascular na família somente $2,7 \%$ dos indivíduos afirmaram ter, sendo que o grupo sem o $\mathrm{FCH}$ apresentou maior percentual $(8,7 \%)$ enquanto o grupo com o $\mathrm{FCH}$ apresentou o percentual de $1,6 \%$.

O histórico de AVC na família dos participantes foi de $16,4 \%$, sendo que nos indivíduos com fenótipo observou-se maior frequência $(17,1 \%)$ em relação aos sem fenótipo foi $13,0 \%$. Somente nas pessoas do grupo com fenótipo verificou-se o histórico familiar de doença renal (1,6\%), assim como a presença de evento de infarto do miocárdio $(8,1 \%)$.

\section{O histórico de}

\section{AVC na família}

dos participantes

foi de $16,4 \%$,

sendo que nos

indivíduos com

fenótipo observou-

se maior frequência

$(17,1 \%)$ em

relação aos sem

fenótipo foi $13,0 \%$.
Na Tabela 3, verificou-se que a estimativa do ritmo de filtração glomerular (eRFG) apresentou média $91,4 \pm 42,6 \mathrm{~mL} /$ min por $1,73 \mathrm{~m}^{2}$ no grupo sem $\mathrm{FCH}$ e no grupo com $\mathrm{FCH}$ a média de $69,3 \pm 23,9$ $\mathrm{mL} / \mathrm{min}$ por $1,73 \mathrm{~m}^{2}$, tendo associação significativa $(\mathrm{p}<0,05)$ para a presença do $\mathrm{FCH}$. Constatou-se também que no grupo com $\mathrm{FCH}, 58$ indivíduos apresentaram eRFG $<60 \mathrm{~mL} / \mathrm{min}$ por $1,73 \mathrm{~m}^{2}$ e no grupo sem FCH 9 indivíduos apresentaram eRFG $<60 \mathrm{~mL} / \mathrm{min}$ por $1,73 \mathrm{~m}^{2}$.

Todas as variáveis antropométricas investigadas tiveram associação com a presença do fenótipo da cintura hipertrigliceridêmica. O IMC de Quetelet apresentou média de $30,6 \pm 5,7 \mathrm{~kg} / \mathrm{m}^{2}$ no grupo com fenótipo e no grupo sem fenótipo $27,4 \pm 4,1 \mathrm{~kg} / \mathrm{m}^{2}$, com alta associação entre as variáveis $(\mathrm{p}<0,05)$. Houve associação significativa também entre a circunferência da cintura (CC) e a presença do fenótipo $(\mathrm{p}<0,001)$, cuja média nos participantes com $\mathrm{FCH}$ foi de $98,2 \pm 9,4 \mathrm{~cm}$. Forte associação também foi encontrada entre a variável relação cintura-estatura (RCE) e FCH ( p <0,001), cuja média foi de $0,631 \pm 0,062 \mathrm{~cm}$ no grupo com fenótipo.

$\mathrm{O}$ índice de conicidade (IC) teve forte relação com a presença do $\mathrm{FCH}(\mathrm{p}<0,05)$, com média de $1,343 \pm 0,085$ no grupo fenotípico e $1,285 \pm 0,113$ para o grupo 


\section{artigo}

Medeiros, T.H.R.S.; Monteiro, S.C.M.; Lima Júnior, J.R.M.; Alves, A.K.B.F.; Sampaio, T.N.S.; Teles, B.E.M.

Fenótipo de cintura hipertrigliceridêmica e lesão renal em pacientes hipertensos

Tabela 3. Relação entre variáveis antropométricas e alteração no Ritmo de Filtração Glomerular em hipertensos assistidos em um centro de prevenção de doença renal. São Luís, Maranhão, 2018.

\section{FENÓTIPO}

VARIÁVEIS

$\begin{array}{cccc}\text { N } & \mathrm{N}=123 & \mathrm{~N}=23 \\ \text { Erfg } & \mathrm{MD} \pm \mathrm{DP} & \mathrm{MD} \pm \mathrm{DP} & \\ \text { PAS } & 69,3 \pm 23,9 & 91,4 \pm 42,6 & 0,004 \\ \text { PAD } & 139,8 \pm 22,8 & 137,3 \pm 22,4 & 0,771 \\ \text { FC } & 82,5 \pm 15,4 & 85,5 \pm 14,6 & 0,380 \\ \text { IMC } & 76 \pm 10,2 & 75,4 \pm 10,8 & 0,792 \\ \text { CC } & 30,6 \pm 5,7 & 27,4 \pm 4,1 & 0,015 \\ \text { RCE } & 98,2 \pm 9,4 & 89,8 \pm 11,2 & 0,000 \\ \text { IC } & 0,631 \pm 0,062 & 0,573 \pm 0,058 & 0,000 \\ \text { IAC } & 1,343 \pm 0,085 & 1,285 \pm 0,113 & 0,005 \\ & 0,766 \pm 0,094 & 0,68 \pm 0,087 & 0,000\end{array}$

eRFG= estimativa de ritmo de filtração glomerular; $P A S$ : pressão arterial sistólica; $P A D=$ pressão arterial diastólica; $F C=$ frequência cardíaca; IMC= Índice de massa corporal; $C C=$ circunferência da cintura; RCE: relação cintura estatura; IC= Índice de conicidade; IAC= Índice de adiposidade central. Fonte: Silva, 2019.

Tabela 4. Relação entre variáveis séricas e alteração no Ritmo de Filtração Glomerular em hipertensos assistidos em um centro de prevenção de doença renal. São Luís, Maranhão, 2018.

\section{FENÓTIPO}

VARIÁVEIS

Erfg

Glicose

Potássio

Ureia

Creatinina

Acido úrico

HDLC

LDLC

Colesterol total

Triglicerídeos

Índice TYG

N HDLC

Ct / HDLC

LDLC/HDLC

IAP

LAP

$\mathrm{Ht}$
SIM

$\mathrm{N}=123$

$\mathrm{MD} \pm \mathrm{DP}$

$69,3 \pm 23,9$

$113,9 \pm 32,1$

$4,3 \pm 0,7$

$30,9 \pm 10,8$

$1,1 \pm 0,3$

$4,9 \pm 1,5$

$47,9 \pm 14,2$

$113,3 \pm 32$

$202,6 \pm 34,6$

$192,1 \pm 66,1$

$2,139 \pm 0,099$

$154,7 \pm 34,2$

$4,71 \pm 3,09$

$2,55 \pm 0,95$

$0,6 \pm 0,2$

$88,7 \pm 42,4$

$40,2 \pm 4,1$

\section{NÃO}

$$
\mathrm{N}=23
$$

$\mathrm{MD} \pm \mathrm{DP}$

$$
91,4 \pm 42,6
$$

$96,9 \pm 13,1$

$5,7 \pm 13,5$

$37,8 \pm 11,9$

$1,2 \pm 2,8$

$5,5 \pm 2,4$

$51 \pm 10,1$

$126,6 \pm 29,2$

$199 \pm 37,4$

$152,6 \pm 129,5$

$2,048 \pm 0,114$

$148 \pm 39,4$

$4,06 \pm 1,07$

$2,54 \pm 0,64$

$0,4 \pm 0,3$

$53,4 \pm 44,1$

$39,4 \pm 4,6$
P

0,0047

0,089

0,745

0,097

0,817

0,313

0,314

0,066

0,660

0,030

0,000

0,405

0,317

0,952

0,000

0,000

0,586 sem alterações. $\mathrm{O}$ índice de adiposidade central (IAC) também demonstrou ter relação com a presença da cintura hipertrigliceridêmica ( $p<0,001)$, as pessoas do grupo com fenótipo apresentaram média 0,766 $\pm 0,094$ e o grupo sem fenótipo, tiveram média de $0,68 \pm 0,087$. A glicemia em jejum não apresentou diferença expressiva $(p=0,089)$ entre os grupos estudados, sendo que no grupo com a característica fenotípica houve maior média da glicemia, com média de $113,9 \pm 32,1 \mathrm{mg} / \mathrm{dL}$ e o grupo de hipertensos sem o fenótipo apresentou média de $96,9 \pm 13,1 \mathrm{mg} / \mathrm{dL}$.

No que diz respeito a pressão arterial, verificou-se que o grupo com fenótipo apresentou maior média de pressão arterial sistólica, com valores de $139,8 \pm 22,8$ $\mathrm{mmHg}$ enquanto o grupo sem fenótipo apresentou média de $137,3 \pm 22,4 \mathrm{mmHg}$, sem diferença significativa $(p=0,771)$. A pressão diastólica apresentou maior média no grupo sem fenótipo $85,5 \pm 14,6$ enquanto a média no grupo sem fenótipo foi de $82,5 \pm 15,4$, sem também, apresentar diferenças estatísticas entre os grupos estudados $(p=0,380)$.

Conforme a Tabela 4 que descreve os parâmetros laboratoriais, os valores plasmáticos de triglicerídeos obtiveram médias de $192,1 \pm 66,1 \mathrm{mg} / \mathrm{dL}$ e de $152,6 \pm 129,5 \mathrm{mg} /$ dL para quem tem a presença do fenótipo e para quem não tem o $\mathrm{FCH}$, respectivamente, e relação com alta significância para o fenótipo $(\mathrm{p}<0,05)$. Os índices de TyG, IAP e o LAP foram altamente significati$\operatorname{vos}(\mathrm{p}<0,001)$ nos participantes do grupo com FCH. Para quem foi classificado com fenótipo, as médias de TyG, IAP e LAP foram 2,139 $\pm 0,099 ; 0,6 \pm 0,2$ e 88,7 $\pm 42,4$, respectivamente. Para quem não teve alteração para o $\mathrm{FCH}$, as médias para os mesmos índices foram um pouco menores 2,048 $\pm 0,114 ; 0,4 \pm 0,3$ e $53,4 \pm 44,{ }^{1}$.

Para as outras variáveis bioquímicas como glicose, LDL-c, HDL-c e colesterol total não foram observados uma relação expressiva, assim como não houve diferença com significância entre colesterol não HDL, relação colesterol total/HDL-c, relação HDL-c/LDL-c e hematócrito e hemoglobina, entre os dois grupos. 
$\mathrm{Hb}$

$13,4 \pm 2,6$

$13,3 \pm 1,5$

0,848

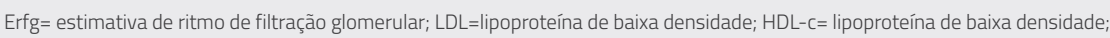
TYG= indice triglicerídeo/ glicose; IAP= índice de aterogenicidade; $L A P=$ lipid accumulation product $\mathrm{Ht}=$ hematócrito $\mathrm{Hb}=\mathrm{Hemo}-$ globina. Fonte: Silva, 2019

Os valores de uréia não demonstrou diferença significativa $(p=0,357)$ entre os respectivos grupos, entretanto, vê-se que no grupo de hipertensos com fenótipo a média foi menor do que no grupo sem fenótipo, com $30,9 \pm 10,8 \mathrm{mg} / \mathrm{dl}$ e $37,8 \pm 11,9 \mathrm{mg} / \mathrm{dl}$, respectivamente. Em relação a creatinina, também não houve diferença estatisticamente significante $(p=0,817)$ sendo que o grupo sem fenótipo apresentou média de $1,2 \pm 2,8 \mathrm{mg} / \mathrm{dL}$ e os com fenótipo apresentaram uma média de $1,1 \pm 0,3 \mathrm{mg} / \mathrm{dL}$.

\section{DISCUSSÃO}

O principal achado do presente estudo foi que o fenótipo da cintura hipertrigleceridêmica está asssociado como um marcador de anormalidades metabólicas nesta população hipertensa ludovicense. A prevalência do $\mathrm{FCH}$ foi elevada $(84,2 \%)$, sendo $35 \%$ homens e $65 \%$ para mulheres, superando dados publicados por outros pesquisadores. Estudo na cidade de São Luís (MA), envolvendo 218 mulheres hipertensas de meia idade, a prevalência encontrada foi de $33 \%^{7}$; por sua vez, um estudo de delineamento longitudinal na cidade de Viçosa (MG), identificou a prevalência do $\mathrm{FCH}$ em $17,2 \%$ dos adultos saudáveis participantes ${ }^{17,18,19}$

Alguns estudos realizados em diferentes regiões do Brasil, utilizando o mesmo ponto de corte da presente pesquisa, mostraram prevalência do fenótipo entre 17 a 20,5\% e investigando adultos e idosos hipertensos na Bahia encontrou prevalência de $26,3 \%$ de fenótipo da cintura hipertrigliceridêmica. Um estudo investigando homens e mulheres pertencentes à população americana, de grupos étnicos distintos, em vários estados, encontrou prevalência de $14,9 \%$ do $\mathrm{FCH}^{20,21}$.

A predominância do $\mathrm{FCH}$ no sexo feminino encontrada no presente estudo condiz com o que foi achado por Freitas (2016) e Oliveira (2014), no ambulatório da Universidade Federal de Viçosa, no

\section{o ponto de corte da IDF para CC, iden-}

Alguns estudos

realizados em

diferentes regiões

do Brasil, utilizando

o mesmo ponto

de corte da presente

pesquisa, mostraram

prevalência do

fenótipo entre

17 a $20,5 \%$ e

investigando adultos

e idosos hipertensos

na Bahia encontrou

prevalência de

$26,3 \%$ de fenótipo

da cintura

hipertrigliceridêmica. qual $56,9 \%$ de indivíduos com fenótipo eram do sexo feminino. Entretanto, outros estudos verificaram predominância do sexo masculino para o fenótipo ${ }^{21}$. Em pesquisa realizada na região Sul do Brasil, com jovens de ambos os sexos a prevalência do fenótipo foi maior em homens, perfazendo $5,9 \%$ e $4,5 \%$ para mulheres e, em pesquisa conduzida no Peru, que utilizou tificou maior proporção de homens com FCH $(38,1 \%)$, se comparado ao que foi encontrado nas mulheres $(30,3 \%)^{22}$.

A constatação de elevada presença do FCH nesses participantes é uma alerta para o cuidado em saúde com os mesmos, uma vez que, constatou-se que os indivíduos com presença de $\mathrm{FCH}$, quando comparados àqueles com circunferência abdominal e níveis de triglicerídeos dentro da normalidade, apresentaram 3,6 vezes mais chances de desenvolver doenças cardiovasculares e complicações coronarianas..

Sabe-se que o $\mathrm{FCH}$ foi proposto como uma ferramenta simples e de baixo custo para identificar indivíduos que apresentam a tríade metabólica aterogênica condição que predispõe os seus portadores ao alto risco para doenças cardiovasculares e metabólicas, tais como a doença renal ${ }^{21}$.

St-Pierre et al. (2007), em estudo longitudinal, mostraram que, além dos indivíduos que tinham o $\mathrm{FCH}$ apresentarem um perfil metabólico favorável a doenças cardiovasculares se comparados àqueles sem o fenótipo, uma proporção importante de homens $(53,4 \%)$ e, principalmente, de mulheres $(78,9 \%)$ com o fenótipo, também apresentavam outras alterações metabólicas. Dessa maneira, vê-se que a presença simultânea da obesidade abdominal e da hipertrigliceridemia - fenótipo da cintura hipertrigliceridêmica - parece predispor o indivíduo a hiperglicemia, alteração da pressão arterial, doenças cardiovasculares, doenças coronárias, obesidade e lesão renal7,19.

$\mathrm{O}$ alto percentual de fenotípicos na terceira idade mostra-se um achado comum. Em estudos na cidade de São Luís verificou-se achados que corroboram com o presente do estudo, no tocante ao predo- 
mínio do fenótipo em pessoas idosas ${ }^{2,23}$. Além disso, as doenças crônicas que impactam no desenvolvimento do fenótipo, tais como hipertensão, diabetes mellitus e obesidade também são predominantes na terceira idade ${ }^{22,23}$.

Com o envelhecimento, a combinação de fatores que vão desde as alterações fisiológicas da própria idade como a mudança na composição corporal, a perda de massa magra e a maior concentração de gordura na região abdominal, até o maior risco metabólico acumulado ao longo da vida, aumentam a taxa de doenças crônicas nessa populaçãa ${ }^{24}$. O envelhecimento modifica o valor preditivo dos fatores de risco tradicionais como, por exemplo, entre os idosos o colesterol total e o LDL-c representam preditores de risco de menor força já que a hipercolesterolemia aumenta com a idade para homens e mulheres, mas o seu valor declina nas faixas etárias mais avançadas ${ }^{23,25}$.

Os riscos se tornam maiores quando há aumento da pressão arterial, como a população investigada no presente estudo. Alguns estudos mostram resultados semelhantes, nos quais os participantes portadores de alterações simultâneas nas concentrações plasmáticas de TG e na medida da CC, apresentaram níveis pressóricos mais elevados que os indivíduos que apresentavam tais parâmetros dentro da normalidade 26,27,29. O Hoorn Study indicou que homens e mulheres cintura hipertrigliceridêmica se encontravam com risco aumentado para doença cardiovascular, mesmo quando apresentavam o metabolismo de glicose dentro dos parâmetros considerados normais ${ }^{23,22}$. Entre as doenças cardiovasculares, constatou-se que a hipertensão arterial sistêmica (HAS), expõe o paciente a maiores chances de desenvolver ou apresentar o $\mathrm{FCH}^{7}$.

Quanto ao baixo percentual de prática de atividade física encontrada na amostra total do estudo, viu-se que na cidade de Pelotas (RS), também identificaram essa prática como uma característica entre os portadores do fenótipo da cintura hipertrigliceridêmica ${ }^{22}$.

Andrade (2017) verificou que a atividade física de intensidade fraca ou ausente foi associada à maior prevalência de $\mathrm{FCH}$. Estudo com idosos comprovou que a atividade física regular estava inversamente associada com a síndrome metabólica e que o risco de obesidade central aumenta com a idade e está fortemente associado ao sedentarismo ${ }^{29}$. Czernicho (2007) verificou que os indivíduos que apresentaram o FCH eram fisicamente inativos e ainda fumavam frequentemente. Entretanto, não foi identificado tabagismo expressivo entre os participantes dessa pesquisa.

\section{Entre as doenças cardiovasculares,}

constatou-se que

a hipertensão

arterial sistêmica

(HAS), expõe

o paciente a

maiores chances

de desenvolver ou

apresentar o FCH.

Quanto as condições de saúde, está sedimentado na literatura que a presença de diabetes mellitus, hipertensão arterial e hipercolesterolemia são fatores intrinsecamente ligados a síndrome metabólica e ao aparecimento do $\mathrm{FCH}^{30,31,25,30,32}$.Assim como foi observado em estudo desenvolvido

O sobrepeso e a obesidade, mensura- dos pelo IMC, se associaram fortemente à $\mathrm{FCH}$, fato demonstrado pela significativa associação com a presença do $\mathrm{FCH}$ nos participantes avaliados. Esse achado está de acordo com o encontrado por Mendes e Melendez (2009), que observaram que $21,4 \%$ de uma população do semiárido de Minas Gerais era obesa e tinha $\mathrm{FCH}$ ( $p<0,001)$ e também por Amini e cols. (2011) que, ao estudarem uma amostra de indivíduos do Irã, verificaram que aqueles com FCH têm maiores IMC ( $\mathrm{p}$ $<0,001)^{2,33}$. Esses autores têm demonstrado que indivíduos com FCH apresentam obesidade global, além da gordura visceral aumentada, fato explicado pela forte correlação da CC com o IMC.

A importância das consequências do excesso de peso é enfatizada em estudo que mostra a relação entre aumento da massa corporal com elevação da pressão arterial, alterações metabólicas e aumento do risco cardiovascular e de doença renal.

Além dos riscos cardiometabólicos, o sobrepeso e a obesidade estão associados a alterações renais hemodinâmicas, estruturais e histológicas, bem como desordens metabólicas e bioquímicas que predispõem à doença renal, mesmo com a função renal estando normal nos exames convencionais. Atualmente, sabe-se que o tecido adiposo não é apenas um reservatório de gordura, mas um tecido dinâmico envolvido na produção de "adipocinas", incluindo a leptina, adiponectina, fator de necrose tumoral- $\alpha$, proteína quimiotática de monócitos-1, fator de transformação do crescimento- $\beta$ e angiotensina-II ${ }^{33}$

Uma série de eventos é desencadeada pela obesidade, incluindo resistência à insulina, intolerância à glicose, hiperlipidemia, aterosclerose e hipertensão arterial, estando todos associados a aumento do risco cardiovascular. A associação entre DRC e dislipidemia também tem sido descrita, mas as causas ainda são desconhecidas. Porém, sabe-se que a resistência à insulina, presente na doença renal crônica, reduz a atividade da lípase lipoproteica, fato este que está intimamente envolvido na fisiopatologia da dislipidemia na doença renal crônica ${ }^{34}$. 
No presente estudo constatou-se forte associação entre o índice TyG (resistência insulínica) e a diminuição da estimativa do ritmo de filtração glomerular com o fenótipo da cintura hipertrigliceridêmica. Estudo realizado com análise em corte transversal e em perspectiva para avaliar a relação de FCH com DRC na população adulta iraniano verificou que na análise transversal e após o controle para idade, nível de tabagismo, nível educacional, estado civil, índice de massa corporal, diabetes e hipertensão, descobriu-se que a presença do fenótipo da cintura hipertrigliceridêmica foi associada com DRC na população feminina. Alguns estudos vêm encontrando resultados semelhantes ${ }^{34,35}$.

Além do aumento da obesidade e sobrepeso e, consequente circunferência abdominal, a dislipidemia é muito comum entre os pacientes com DRC. O perfil lipídico varia muito nestes pacientes, refletindo o nível de função renal e do grau de proteinúria. Em geral, o grau de hipertrigliceridemia é proporcional à gravidade da insuficiência renal.

No presente estudo a hipertrigliceridemia foi um fator de associação entre os participantes com a presença do fenótipo. No estudo de Zhe (2012) o fenótipo cintura hipertrigliceridêmica esteve associado a uma carótida aterosclerótica pior na população de estudo, mesmo usando um valor de corte mais rigoroso para a obesidade abdominal. Isto indica que o fenótipo da cintura hipertrigliceridêmica podem ser úteis para prever o risco de DCV em pacientes com DRC 34,36 .
O índice antropométrico relação cintura estatura (RCE) demonstrou, no presente estudo uma associação com o FCH nos participantes. Corroborando com esses resultados, uma metanálise que demonstrou que RCE poderia ser superior a circunferência da cintura e ao IMC na avaliação da obesidade central e o risco de ocorrência de alterações metabólicas nos indivíduos.

Além disso, um estudo de corte transversal realizado em 4611 participantes, mostrou que RCE foi significativamente associado com DRC, independentemente da hipertensão e diabetes. Assim, esse parâmetro pode ser utilizado também para avaliação de obesidade central e, consequentemente para avaliação do risco de lesão renal em pacientes com o $\mathrm{FCH}^{29,35,36}$.

Embora muitos estudos tentem esclarecer a associação entre índices antropométricos e doença renal crônica, estes estudos diferem um do outro em termos de diferentes tipos de modelos de estudo, parâmetros de classificação antropométricos e tempos de seguimento diferenciados. Mesmo assim, existem fatores de risco muito bem sedimentados na literatura para o desenvolvimento e progressão da DRC tais como a idade, o sexo feminino, hipertensão arterial, diabetes mellitus, índices antropométricos, tabagismo e dislipidemia, principalmente a hipertrigliceridemia isolada.

A doença renal está associada a várias complicações tais como anemia, acidose metabólica (redução da excreção de ácido pelos rins) e doença cardiovascular, o que aumenta a complexidade do controle e tra- tamento do doente, além do impacto econômico e social. Sabe-se que pacientes hipertensos são grupo de risco clássico para o aparecimento da lesão renal e progressão da doença de forma crônica e essa patologia pode estar presente nos primeiros estágios da DRC e está bem documentado para contribuir para a morbidade cardiovascular 36,37,38,39.

Logo, o acúmulo de fatores de risco pode levar ao aumento da probabilidade do aparecimento da lesão renal e consequente progressão para doença renal crônica, o rastreamento pelo fenótipo da cintura hipertrigliceridêmica tem potencial como ferramenta de triagem para identificar indivíduos com risco de insuficiência renal.

\section{CONCLUSÃO}

No presente estudo, o fenótipo da cintura hipertrigliceridêmica, além de oferecer alterações antropométricas e metabólicas, pareceu expor também os indivíduos hipertensos à lesão renal mais precocemente quando comparado àqueles que não apresentaram o fenótipo, corroborando com alguns estudos com a mesma temática.

Entretanto, para adotar esse critério como fator de risco para doença renal crônica, existe a necessidade de estudos com maior representatividade populacional e de abordagem longitudinal assim como a definição de pontos de corte específicos para a população brasileira e para os diferentes ciclos de vida a fim de descartar outros possíveis fatores.m

\section{REFERÊNCIAS}

1. Schrier R W et al. Insuficiência Renal Aguda e Sepse. New England Journal of Medicine.2004; 351 (2):159-169.

2. Mendes M S F; Melendez J G V. Cintura hipertrigliceridêmica e sua associação com fatores de risco metabólicos [Dissertação]. Belo Horizonte: Universidade Federal de Minas Gerais; 2009.

3. Brasil. Ministério da Saúde. Secretaria de Atenção à Saúde. Departamento de Atenção Especializada e Temática. Diretrizes Clínicas para o Cuidado ao paciente com Doença Renal Crônica - DRC no Sistema Único de Saúde/ Ministério da Saúde [livro eletrônico] Brasília, 2014. ISBN 1. [acesso em outro de 2018]. Disponível em
4. Seymen P. et al. Evaluation of visual evoked potentials in chronic renal failure patients with diferente treatment modalities. Journal of Nephrology, sem cidade.2010;v. 23, n. 06:705-710.

5. Marinho A G B et al. Prevalência de doença renal crônica em adultos no Brasil: revisão sistemática da literatura. Cad. Saúde Colet. $2017 ; 25$ (3): 379-388.

6. Santos F M J R et al .2017. Estratégias da atenção básica na doença renal crônica: a importância do diagnóstico precoce. Rev. Saúde.Com. 2017. 13(2), 863-870. 


\section{REFERÊNCIAS}

7. CabraL N A L et al. Hypertriglyceridemic waist and cardiometabolicrisk in hypertensive women.Rev Assoc Med Bras. 2012. 58:568-73.

8. Macinko J; Harris M J. Brazil's family health strategy-delivering community-based primary care in a universal health system. $\mathrm{N}$ Engl J Med. 2015; 372:2177-81.

9. Lemieux I P A et al. Hypertriglyceridemic waist: A marker of the atherogenic metabolic triad (hyperinsulinemia; hyperapolipoprotein B; small, dense LDL) in men?. Circulation. 2000;102(2):179-84.

10. Cardinal T R. Pontos de corte ótimos para a circunferência da cintura e relação cintura-quadril na definição da síndrome metabólica no brasil - estudo longitudinal de saúde do adulto (elsa-brasil). [tese de doutorado]. Porto Alegre. Universidade Federal do Rio Grande do Sul. 2015.

11. Romagnani P., Remuzzi G., Glassock R. et al. Chronic kidney disease. Nat Rev Dis Primers 3. 2017.17088. https://doi. org/10.1038/nrdp.2017.88

12. Haskell W L. Physical Activity and Public Health: Updated Recommendation for Adults from the American College of Sports Medicine and the American Heart Association. Medicine \& Science in Sports \& Exercise.2007; 39(8):1423-1434.

13. IBGE. Pesquisa Nacional de Saúde 2013: percepção do estado de saúde, estilos de vida e doenças crônicas. Brasil, grandes regiões e unidades da federação. Rio de Janeiro: IBGE; 2014. Disponivel em: http://www.ibge.gov.br/home/estatistica/populacao/ pns/2013.

14. Brasil. Ministério da Saúde. Secretaria de Vigilância em Saúde. Departamento de Doenças e Agravos não transmissíveis e Promoção da Saúde. Vigilância de Fatores de Risco e Proteção para Doenças Crônicas por Inquérito Telefônico, Vigitel 2014. Brasília: Ministério da Saúde 2015.

15. International diabetes federation. Idf atlas. 7th ed. Brussels, belgium: international diabetes federation; 2015.

16. Sociedade brasileira de cardiologia. Departamento de hipertensão arterial. VI Diretrizes brasileiras de hipertensão. Rev Bras Hipertens. 2017;17(1):4-62

17. Santos M A S et al. Tendências da morbidade hospitalar por doenças crônicas não transmissiveis no Brasil, 2002 a 2012. Epidemiol e Serviços Saúde. 2015;24(3):398-389.

18. Oliveira C C R et al. Hypertriglyceridemic waist phenotype: association with metabolic disorders and visceral fat in adults. Nutr Hosp 2014; 30:25-31.

19. Hanley, A.J. Prediction of type 2 diabetes mellitus with alternative definitions of the metabolic syndrome: the Insulin Resistance Atherosclerosis Study. Circulation 2005; 112: 3713- 3721.

20. Mota A P. Cintura Hipertrigliceridêmica em Pacientes Hipertensos. International Journal of Cardiovascular Sciences. 2016;29(3):175-180.

21. Haack R L et al. The hypertriglyceridemic waist phenotype in young adults from the Southern Region of Brazil. Cad Saúde Pública. 2013;29(5):999-1007.
22. Fagundes A C G et al. Avaliação da prevalência de idosos polifarmácia pelo Programa PET Saúde em Estratégia de Saúde da Família. Rev. Saúde Coletiva. 2020; (10) N.57.

23. Gomez-Huelgas R et al. Hypertriglyceridemic waist: an alternative to the metabolic syndrome? Results of the IMAP Study (multidisciplinary intervention in primary care). Int J Obes (Lond). 2011;35(2):2929.

24. CABRAL R A L et al. Hypertriglyceridemic waist phenotype and cardiometabolic alterations in Brazilian adults. Nutr Hosp 2015; 32:1099-106.

25. BARRETO S M et al. Chronic kidney disease among adult participants of the ELSA-Brasil cohort: association with race and socioeconomic position. J Epidemiol Community Health. 2016;70(4):380-9.

26. Rim, J H L Y, Cha; B , Lee S, Kim J. Central obesity is an independent risk factor for microalbuminuria in both the general Korean women and nondiabetic nonhypertensive subpopulation: Association of microalbuminuria and metabolic syndrome from the Korea National Health and Nutrition Examination Survey 20112012.Clinica Chimica Acta .2015. 448: 74-79

27. LI Y et al. Hypertriglyceridemic Waist Phenotype and Chronic Kidney Disease in a Chinese Population Aged 40 Years and Older. March 2014. (9).

28. Glassock, R.J; Winearls C. The global burden of chronic kidney disease: how valid are the estimates?. Nephron Clin Pract. 2008;110(1):c39-47.52

29. Freitas, R.S et al. Fenótipo cintura hipertrigliceridêmica: fatores associados e comparação com outros indicadores de risco cardiovascular e metabólico no ELSA-Brasil. Cad. Saúde Pública 2018; 34(4).

30. Strasser B et al. Obesidade visceral e resposta inflamatória: uma revisão das evidências. Obes Rev 2012; 7: 578-91.

31. Yu D, et al. Hypertriglyceridemic-waist is more predictive of abnormal liver and renal function in an Australian population than a Chinese population. Obesity. Res Clin Pract.2018.

32. Barzin $\mathrm{M}$ et al. Changes in waist circunference and incidence of chronic kidney disease. Eur J Clin Invest. 2014; 44 (5): 470-476

33. Declèves A E; Sharma, K. Obesity and kidney disease: differential effects of obesity on adipose tissue and kidney inflammation and fibrosis. Curr Opin Nephrol Hypertens. 2015;24:28-36. Ramezankhani $A$, etal. The hypertriglyceridemic waist and waistto-height ratio phenotypes and chronic kidney disease: Cross sectional and prospective investigations.Obes Res.Clin. Pract .2016.

34. Zhe $X$ et al. Hypertriglyceridemic Waist is Associated with Increased Carotid Atherosclerosis in Chronic Kidney Disease Patients. Nephron Clin Pract. 2012;122:146-152.

35. Martins J R. Perfil clínico e epidemiológico da doença renal crônica: revisão integrativa. 2017.[Trabalho de Conclusão de Curso]. São luís. Universidade Federal do Maranhão.

36. Ji $B$ et al.The risk factors of mild decline in estimated glomerular filtration rate in a community-based population. Clinical Biochemistry. 2013; 46: 750-754. 